\title{
Azorhizobium caulinodans ORS571 Colonizes the Xylem of Arabidopsis thaliana
}

\author{
Philip J. Stone, Kenneth J. O’Callaghan, Michael R. Davey, and Edward C. Cocking \\ Centre for Crop Nitrogen Fixation, Plant Science Division, School of Biosciences, University of Nottingham, \\ Nottingham NG7 2RD, U.K. \\ Accepted 18 September 2000.
}

Improved conditions were used for the aseptic growth of Arabidopsis thaliana to investigate whether xylem colonization of A. thaliana by Azorhizobium caulinodans ORS571 might occur. When seedlings were inoculated with ORS571 (pXLGD4) tagged with the lac Z reporter gene, nearly all of the plants showed blue regions of ORS571 colonization at lateral root cracks (LRC). The flavonoids naringenin and liquiritigenin significantly stimulated colonization of LRC by ORS571. Blue bands of ORS571 (pXLGD4) bacteria were observed histochemically in the xylem of intact roots of inoculated plants. Detailed microscopic analysis of sections of primary and lateral roots from inoculated $A$. thaliana confirmed xylem colonization. Xylem colonization also occurred with an ORS571 nodC mutant deficient in nodulation factors. There was no significant difference in the percentage of plants with xylem colonization or in the mean length of xylem colonized per plant between plants inoculated with either ORS571 (pXLGD4) or ORS571::nodC (pXLGD4), with or without naringenin.

Additional keywords: nitrogen fixation.

Arabidopsis thaliana has become a widely accepted model plant for the study of plant-pathogen interactions (Crute et al. 1994; Dangl 1993). Recently A. thaliana has been suggested as a model nonlegume for the study of root interactions with diazotrophic bacteria such as Azorhizobium caulinodans ORS571 (Gough et al. 1996). An advantage of A. thaliana is the simplicity of the organization of its root system, its largely invariant ontogeny, and its fixed cellular organization (Scheres and Wolkenfelt 1998). The colonization by ORS571 of cracks at the sites of $A$. thaliana lateral root emergence and the invasion of intercellular spaces between cortical and endodermal cell layers was observed, but xylem colonization was not detected (Gough et al. 1997a). Xylem colonization in the roots of A. thaliana (Deslandes et al. 1998) and tomato (Vasse et al. 1995) plants inoculated with the bacterial wilt pathogen Ralstonia solanacearum, however, has been observed. Xylem colonization was also detected in the roots of tomato plants

Corresponding author: E. C. Cocking; Telephone: +44 115 9513056; Fax: +44 115 9513240; E-mail: edward.cocking@ nottingham.ac.uk inoculated with a $R$. solanacearum $\mathrm{HrcV}^{-}$mutant that is unable to elicit a hypersensitive response or induce wilting in susceptible hosts such as tomato (Etchebar et al. 1998). These observations, together with the finding that ORS571 colonizes root xylem elements (O'Callaghan et al. 1997) and induces nitrogen-fixing nodules in the root cortex of the legume Sesbania rostrata, led us to reinvestigate whether xylem colonization of $A$. thaliana roots by ORS571 might occur. Recently, agrobacteria were detected in the xylem of $A$. thaliana roots cocultivated with an Agrobacterium strain (De Buck et al. 2000).

A. thaliana (ecotype Col-0) seeds were surface sterilized in 15\% Domestos bleach (Lever Industrial Ltd., Runcorn, U.K.) for $15 \mathrm{~min}$ and grown aseptically under different conditions. Plants were grown in tubes $(25 \times 150 \mathrm{~mm}$ containing $20 \mathrm{ml}$ of medium) or in jars (175-ml capacity containing $50 \mathrm{ml}$ of medium), with or without vented lids. Use of the vented lids improved gas exchange and minimized condensation. The vented lids had 24-mm diameter holes closed with foam bungs $(50 \times$ $37 \mathrm{~mm}$ in diameter) or foil Sun Caps (Sigma, St. Louis, MO, U.S.A.) with $0.02-\mu \mathrm{m}$ filter disks $(10 \mathrm{~mm}$ in diameter). The growth media (with $0.8 \%$ agar wt/vol) were either Murashige and Skoog (MS) (1962) (Sigma M5519) with 0, 1, 3, or 5\% sucrose or Fåhraeus (1957) without sucrose and with $0,0.25$, or $1 \mathrm{mM} \mathrm{KNO}_{3}$. Plants were incubated at $22^{\circ} \mathrm{C}$ during the day $\left(18.5 \mu \mathrm{Em}^{-2} \mathrm{~s}^{-1}\right.$ "daylight" fluorescence for $\left.16 \mathrm{~h}\right)$ and $20^{\circ} \mathrm{C}$ at night. Root and shoot growth were assessed after 4 weeks. The most extensive root and dark-green shoot growth was in the MS medium with $3 \%$ sucrose in jars with vented lids.

With the use of these improved nutritional and environmental conditions for A. thaliana growth, we investigated whether xylem colonization by ORS571 and nodC minus ORS571 would occur. We first determined the percentage of plants with lateral root cracks (LRC) that were colonized by ORS571. In addition, we assessed whether there would be a flavonoid-stimulated increase in LRC colonization. A. caulinodans ORS571 carrying pXLGD4 was cultured on TGYE medium (Ladha et al. 1989) that was semisolidified with $0.8 \%$ (wt/vol) agar and $10 \mu \mathrm{g}$ of tetracycline per milliliter. ORS571::nodC (pXLGD4) (Geelen et al. 1993) was cultured as it had been for ORS571 (pXLGD4) but included $25 \mu \mathrm{g}$ of kanamycin per milliliter. ORS571 (pXLGD4), tagged with a constitutively expressed lacZ reporter gene (Leong et al. 1985), was visualized by the blue pigment resulting from the 
degradation of X-Gal (5-bromo-4-chloro-3-indolyl- $\beta$-Dgalactopyranoside) by the lacZ-encoded $\beta$-galactosidase (Boivin et al. 1990). The endogenous plant $\beta$-galactosidase was first inactivated by fixation in $2 \%$ ( $\mathrm{vol} / \mathrm{vol}$ ) glutaraldehyde for $4 \mathrm{~h}$ (Vasse et al. 1995). The flavonoid naringenin (Sigma) and liquiritigenin (Apin Chemicals Ltd., Abingdon, U.K.) were dissolved, as previously described (Gough et al. 1997a). Four days after surface-sterilized A. thaliana Col-0 seeds had been placed in vented jars on the surface of MS medium (3\% sucrose), with or without added flavonoids $(5 \times$ $\left.10^{-5} \mathrm{M}\right)$, they were inoculated with $1 \mathrm{ml}$ of ORS571 (pXLGD4) $\left(10^{8} / \mathrm{ml}\right.$ in water).

After 4 weeks growth in MS medium with 3\% sucrose, with or without added flavonoids, uninoculated and inoculated plants were examined after fixation and histochemical staining with X-Gal. Inoculated, histochemically stained roots were found to be colonized inside LRC, suggesting that LRC were providing points of entry into the root system for ORS571. Microscopic examination of colonized LRC sections sometimes revealed thickened plant cell walls adjacent to invading bacteria, possibly a manifestation of plant defense. At up to 24 $\mathrm{h}$ after inoculation of 4-week-old plants, however, signs of a hypersensitive response around LRC were not detected after staining pieces of unfixed roots with potassium iodide (Olson and Varner 1993), diaminobenzidine, or 4,6-diamino-2phenylindole (Alvarez et al. 1998). Between 92 and 100\% of A. thaliana plants had some secondary LRC colonized by ORS571 (pXLGD4). Uninoculated plants showed no LRC colonization by bacteria. The mean percentage of secondary LRC colonized by ORS571 (pXLGD4) per plant in the controls was comparable to that observed by Gough et al. (1997a), who grew plants in tubes (four plants per tube) containing Fåhraeus medium with $0.25 \mathrm{mM} \mathrm{KNO}_{3}$ for 10 days prior to inoculation with ORS571 (pXLGD4) and before further growth for 7 days. In naringenin-treated plants, a $35.3 \pm$ $10.9 \%$ (mean \pm standard deviation, $n=12$ ) of LRC were colonized, significantly more $(t$ test $P<0.001)$ than the controls $(9.5 \pm 3.3 \%)$. In an independent experiment, liquiritigenin-treated plants showed $27.7 \pm 12.4 \%(n=16)$ of LRC, which again was colonized significantly more $(P=0.004)$ than the controls $(14.5 \pm 11.3 \% ; n=16)$. Several of the secondary laterals of some inoculated plants were markedly shorter than other roots on the same plant and apparently shorter than laterals emerging from a corresponding position on the primary root of the control plants. Nevertheless, statistical assessments showed that this effect does not indicate a major change in the development of the A. thaliana root system. Numerous independent experiments revealed that the number of secondary laterals has little or no association with the number of LRC colonies of azorhizobia, these two factors typically showing very weak correlation coefficients $(r=-0.22$ to 0.19$)$. These correlation assessments showed that either one of these two factors could explain no more than a few percent of the data variation for the other factor. Furthermore, the mean number of secondary laterals did not differ between the control $(23.0 \pm 10.4 ; n=40)$ and naringenintreated $(22.1 \pm 7.1 ; n=24)$ plants $(P=0.669)$ or between the control $(16.3 \pm 10.0 ; n=16)$ and liquiritigenin-treated $(12.8 \pm 4.3 ; n=16)$ plants $(P=0.22)$.

The mechanisms responsible for stimulation of LRC colonization by flavonoids are presently unclear. Flavonoids in- duce the expression of rhizobial genes with unknown functions, which show no homology to nodulation (Nod) genes (Perret et al. 1994). Flavonoids induce resistance in certain rhizobia to the soybean phytoalexin glyceollin (Parniske et al. 1991). An advantage to using A. thaliana to decipher responsible mechanisms is that numerous mutants have been characterized and the role of plant flavonoids in bacterial colonization can be studied with the mutants of phenylpropanoid and flavonoid pathways (Graham 1998).

During our examination of histochemically stained A. thaliana root systems for LRC colonization, we observed thin, blue bands of xylem in some of the inoculated plants (Fig. 1A). To confirm that these blue bands resulted from $\beta$ galactosidase activity of bacteria within the xylem, samples of $\mathrm{X}$-Gal-treated roots with blue bands were dehydrated in an ethanol series and embedded in LR White resin (Davey et al. 1993). Light microscopic examination of approximately $1-\mu \mathrm{m}$ sections showed blue histochemically stained material (Fig. 1B) and toluidine blue-stained bacterialike material (Fig. 1C) present within the xylem of the central vascular system. Transmission electron microscopy of ultrathin sections that were poststained with lead citrate (Reynolds 1963) confirmed that bacteria were present (Fig 1D) in the xylem of the central vascular system that contained blue bands. In an inoculated plant in which the xylem of the primary root was extensively colonized by ORS571 (pXLGD4), colonization of the xylem of the vascular system of the stem also was visible microscopically by direct examination of the X-Gal-treated stem (Fig. 1E). This was confirmed by the detection of toluidine blue-stained bacterialike material in the xylem of a section of the stem (Fig. 1F). No xylem colonization was observed in uninoculated control plants. This LRC and xylem colonization by ORS571 has features in common with LRC and xylem colonization of tomato seedlings by the pathogen $R$. solanacearum (Vasse et al. 1995). In order to invade the vascular cylinder of tomato seedlings, $R$. solanacearum targets sites where the endodermis is either incompletely differentiated at root-tip extremities or is reorientated at secondary root axils by the outgrowth and development of lateral roots. It seems likely that ORS571 in its pathway to xylem invasion in $A$. thaliana also exploits these sites where the endodermal physical barrier is incompletely differentiated or reorientated.

Because we were able to detect and locate blue regions of histochemically stained plants by direct microscopic examination (Fig. 1A), we were readily able to measure the lengths of colonized xylem in the primary and lateral roots. Colonized xylem regions (Fig. 1A) were photographed sequentially along their lengths. The length of each region, irrespective of width, was measured on the photographs with a rotating wheel map measurer, and the total number of arbitrary units of colonized xylem for each root system was recorded. Conversion from arbitrary units to actual length was based on photographs of graticule slides taken at equivalent magnifications. The total length of the colonized xylem (in primary and lateral roots) was thus determined. Uninoculated plants were routinely included as controls. Small zones of endogenous plant $\beta$-galactosidase activity were evident in regions of the xylem after X-Gal treatment (despite fixation in glutaraldehyde), with blue precipitate forming at the bases and between the base and the tip of some laterals. Within any plant showing these blue zones, the latter occurred at similar positions in 
several laterals, indicating their endogenous nature. Microscopy of sections confirmed the absence of bacteria in these zones. Tissues containing blue bands longer than endogenous blue re- gions were excised and squashed under a cover slip with highmagnification microscopy confirming xylem with bacteria. In two independent experiments, the frequency and length of

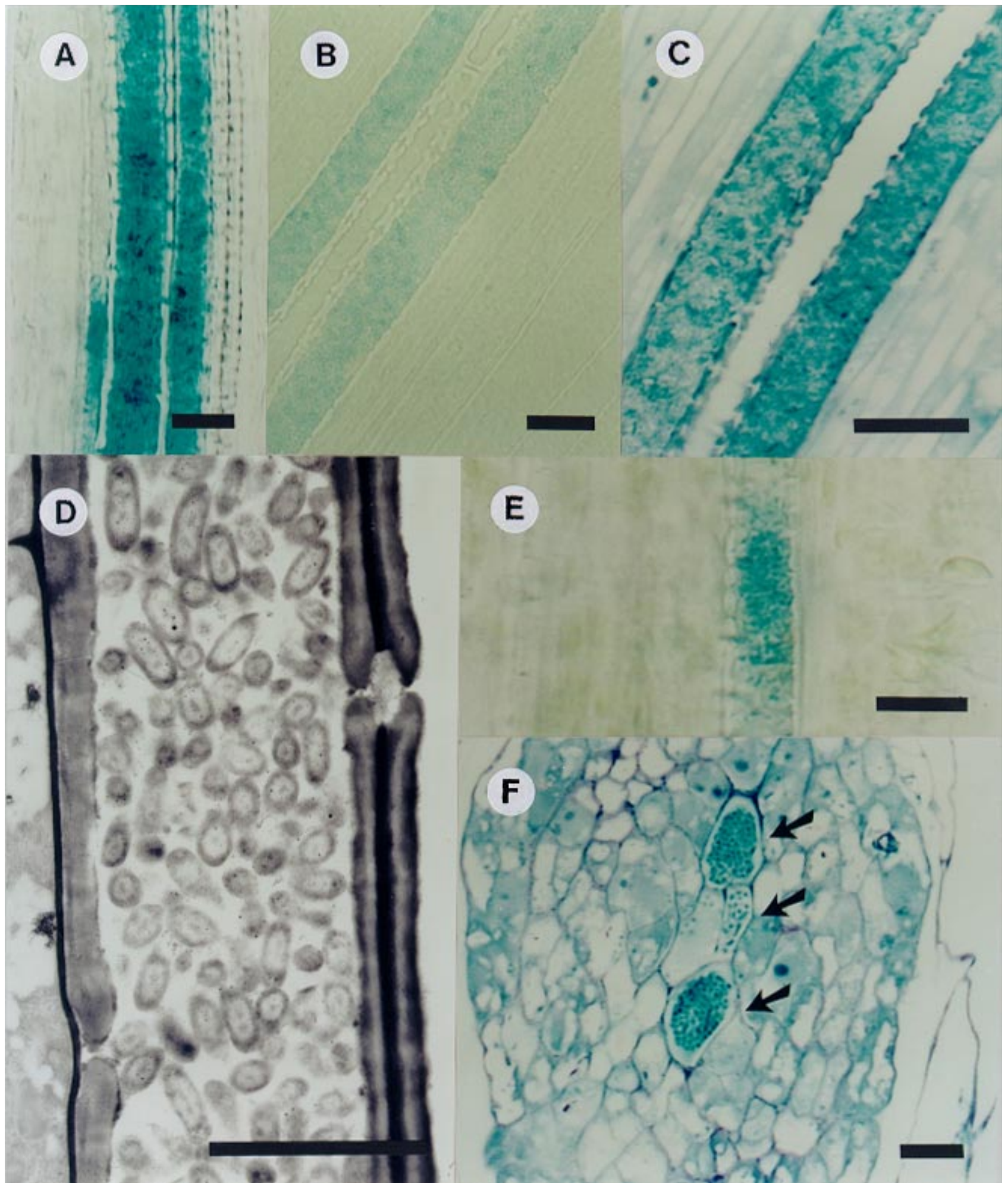

Fig. 1. Colonization of the Arabidopsis thaliana xylem at 4 weeks after inoculation with ORS571 (pXLGD4). A, Primary intact root treated with X-Gal (direct observation) showing blue bands in three regions of the xylem. B, Section of a root showing blue histochemically stained material in the xylem. C, Section of a root (as in B) showing toluidine blue-stained bacterialike material in the xylem. D, Electron micrograph of root xylem (as in C) showing bacteria in a xylem element. E, Intact stem treated with X-Gal (direct observation) showing a blue band in the xylem. F, Transverse section of stem (as in E) showing toluidine blue-stained bacterialike material in the xylem. Bars $=10 \mu \mathrm{m}(\mathrm{A}, \mathrm{B}, \mathrm{C}, \mathrm{E}$, and F) and $5 \mu \mathrm{m}$ (D). 
colonized xylem was determined in plants inoculated with ORS571 (pXLGD4) or ORS571::nodC (pXLGD4), with and without naringenin (Table 1).

Interestingly, LRC colonization of A thaliana by ORS571 is stimulated by flavonoids (Gough et al. 1997a; this study), whereas xylem colonization is not stimulated by naringenin (Table 1). Gough et al (1997a) found that histochemically stained roots of $A$. thaliana inoculated with ORS571 (pXLGD4) were colonized on the surfaces of root tips and inside LRC. Root-tip colonization, however, was not studied subsequently because it was less intense and less reproducible than LRC colonization. There is the possibility that crack entry at root tips is not stimulated by flavonoids and is the major entry port into root xylem. Another possibility is that xylem colonization is initiated only at a small number of (or a single) LRC site(s) and therefore is not stimulated by increases in the percentage of LRC colonized per plant. Future studies of the infection pathway into xylem and the plant development stages important for xylem colonization will probably benefit from the use of root and xylem developmental mutants of $A$. thaliana.

Inoculation with ORS571::nodC (pXLGD4) shows that xylem colonization by ORS571 is Nod-factor independent (Table 1). Colonization of xylem in A. thaliana and tomato by $R$. solanacearum (Deslandes et al. 1998; Vasse et al. 1995) and in sugar cane by the diazotroph Acetobacter diazotrophicus (James et al. 1994) has been observed. Neither of these two bacterial strains produces Nod factors.

In these experiments, we detected no significant differences in the mean number of secondary lateral roots between xylemcolonized plants $(24.3 \pm 8.4 ; n=10)$ and plants with no xylem colonization $(22.4 \pm 9.5 ; n=54)$ or between plants inoculated with ORS571::nodC (pXLGD4) (23.0 $\pm 9.6 ; n=50)$ or ORS571 (pXLGD4) $(21.6 \pm 8.1 ; n=14)$. This noneffect of xylem-located ORS571 on the growth of A. thaliana appears comparable to the reported $R$. solanacearum $\mathrm{HrCV}^{-}$mutant infection of tomato root xylem tissues (Etchebar et al. 1998).

Colonization of the xylem of sorghum leaves by a species of nitrogen-fixing Herbaspirillum has also been observed (James et al. 1997). Recently, we showed that ORS571 in the presence of $5 \times 10^{-5} \mathrm{M}$ naringenin colonized the xylem of the roots of rice (Gopalaswamy et al. 2000). Xylem colonization in rice by ORS571 (pXLGD4) was observed only in the presence of naringenin, in contrast with the situation in A. thaliana. Rice seedlings, however, were grown in Fåhraeus medium rather than the MS medium used for A. thaliana. Bacteria presumed to be $A$. caulinodans have been observed in the xylem of wheat roots repeatedly inoculated with ORS571 (Sabry et al. 1997).

To establish that bacteria in xylem were the strain of $A$. caulinodans used for inoculation, A. thaliana seeds and uninoculated $A$. thaliana plants were first checked for the presence of endophytic bacteria. Whole uninoculated root systems $(n=3)$ and seeds (approximately 500) were surface sterilized (O'Callaghan et al. 1997), macerated, serially diluted, and plated onto TGYE, YEM (Somasegaran and Hoben 1994), Luria-Bertani, peptone broth, and Pseudomonas syringeae-selective (Atlas 1997) agar media before incubation at $25^{\circ} \mathrm{C}$ for 2 to 4 days. No bacteria could be isolated from surface-sterilized seeds and surface-sterilized uninoculated root systems. Bacteria reisolated from plant roots inoculated with A. caulinodans ORS571 (pXLGD4) showed colony morphology characteristic for that strain. Resistant colonies were observed on media containing $10 \mu \mathrm{g}$ of tetracycline per milliliter, and blue colonies were seen on media containing $0.5 \%$ (vol/vol) of $2 \%$ (wt/vol) X-Gal in dimethyl formamide. Colonies on media containing Congo Red did not take up the dye, which is indicative of rhizobia (O'Callaghan et al. 1997). Bacteria isolated from surface-sterilized, previously inoculated roots displayed the same characteristics, but there was a reduction in the number of colony-forming units, approximately $10^{4}$ per plant compared with approximately $10^{6}$ per plant for non-surface-sterilized material.

Previously it was demonstrated with ORS571 carrying a nifD::lacZ fusion that conditions within the intercellular spaces of LRC of A. thaliana (Gough et al. 1996; Gough et al. 1997b) and wheat (Webster et al. 1997) were suitable for nitrogen fixation. Additionally, ORS571-inoculated wheat plants grown in pots exhibited high levels of acetylenereduction activity and greater dry weight and total nitrogen than the control uninoculated plants (Sabry et al. 1997). We have begun to investigate whether conditions in the A. thaliana xylem colonized by nifD::lacZ ORS571 are suitable for nif gene expression. In these studies, unambiguous evidence for nif expression has not been found in plants previously inoculated with a nifD::lacZ ORS571 strain prior to histochemical staining for blue bands of xylem. Our assay procedure, however, does not readily detect small blue regions of colonized xylem, and nif gene expression is likely to be inactivated by the high levels of nitrate $(39 \mathrm{mM})$ and ammonium $(21 \mathrm{mM})$ present in the MS medium. Evidence for nif expression was not found in plants rinsed $24 \mathrm{~h}$ in water prior to staining, but residual nitrates and ammonium might not have been removed effectively from tissues. The use of reporter genes other than $l a c Z$ may permit unambiguous detection of small regions of bacterial nitrogenase activity in inoculated $A$. thaliana. In rice inoculated with Azoarcus sp. strain BH72, transcriptional fusions of gusA and $g f p$ to the nifH gene and immunogold labeling of nitrogenase have revealed high nitrogenase gene expression levels by intercellular Azoarcus sp. in the cortex (Egener et al. 1999).

Table 1. Frequency and length of xylem colonization (XC) in Arabidopsis thaliana inoculated with lacZ reporter strains of azorhizobia and grown with or without $5 \times 10^{-5} \mathrm{M}$ naringenin (data from two independent experiments)

\begin{tabular}{|c|c|c|c|c|c|}
\hline Azorhizobial strain & Naringenin & Plants with $\mathrm{XC}(\%)^{\mathrm{a}}$ & $\mathbf{n}$ & Mean length of XC per plant $(\mu \mathrm{m})^{\mathrm{b}}$ & $n$ \\
\hline ORS571::nodC & No & 8.9 & 45 & 2,501 & 5 \\
\hline ORS571::nodC & Yes & 9.3 & 43 & 2,158 & 4 \\
\hline ORS571 & No & 9.1 & 66 & 3,317 & 6 \\
\hline ORS571 & Yes & 12.1 & 58 & 4,436 & 7 \\
\hline
\end{tabular}

${ }^{\text {a }}$ Chi-square gave no significant difference $(P=0.935)$.

b Analysis of variance showed no significant difference $(P=0.750)$. 


\section{ACKNOWLEDGMENTS}

P. J. Stone and K. J. O'Callaghan were supported by the U.K. Ministry of Agriculture Fisheries and Food. K. J. O'Callaghan also received support from the Samuel Roberts Noble Foundation Inc., and E. C. Cocking received a Leverhulme Trust Fellowship. The authors thank J. Dénarié and colleagues (INRA-CNRS, Toulouse, France) for ORS571 (pXLGD4) and ORS571::nodC (pXLGD4), J. Jones for technical assistance, B. Case for photographic assistance, and R. Dixon of the Samuel Roberts Noble Foundation for helpful suggestions.

\section{LITERATURE CITED}

Alvarez, M. E., Pennell, R. I., Meijer, P. J., Ishikawa, A., Dixon, R. A., and Lamb, C. 1998. Reactive oxygen intermediates mediate a systemic signal network in the establishment of plant immunity. Cell 92:773-784.

Atlas, R. M. 1997. Handbook of Microbiological Media. CRC Press, London.

Boivin, C., Camut, S., Malpica, C. A., Truchet, G., and Rosenberg, C. 1990. Rhizobium meliloti genes encoding catabolism of trigonelline are induced under symbiotic conditions. Plant Cell 2:1157-1170.

Crute, I., Beynon, J., Dangl, J., Holub, E., Mauch-Mani, B., Slusarenko, A., Staskawicz, B., and Ausubel, F. 1994. Microbial pathogenesis of Arabidopsis. Pages 705-747 in: Arabidopsis. E. M. Meyerowitz and C. R. Sommerville, eds. Cold Spring Harbor Laboratory, Cold Spring Harbor, NY, U.S.A.

Dangl, J. L. 1993. The emergence of Arabidopsis thaliana as a model for plant-pathogen interactions. Adv. Plant Pathol. 10:127-156.

Davey, M. R., Webster, G., Manders, G., Ringrose, F. L., Power, J. B., and Cocking, E. C. 1993. Effective nodulation of micro-propagated shoots of the non-legume Parasponia andersonii by Bradyrhizobium. J. Exp. Bot. 44:863-867.

De Buck, S., De Wilde, C., Van Montagu, M., and Depicker, A. 2000. Determination of the T-DNA transfer and the T-DNA integration frequencies upon cocultivation of Arabidopsis thaliana root explants. Mol. Plant-Microbe. Interact. 13:658-665.

Deslandes, L., Pileur, F., Liaubet, L., Camut, S., Can, C., Williams, K., Holub, E., Beynon, J., Arlat, M., and Marco, Y. 1998. Genetic characterization of RRS1, a recessive locus in Arabidopsis thaliana that confers resistance to the bacterial soilborne pathogen Ralstonia solanacearum. Mol. Plant-Microbe Interact. 11:659-667.

Egener, T., Hurek, T., and Reinhold-Hurek, B. 1999. Endophytic expression of nif genes of Azoarcus sp. strain BH72 in rice roots. Mol. PlantMicrobe Interact. 12:813-819.

Etchebar, C., Trigalet-Demery, D., Van Gijsegem, F., Vasse, J., and Trigalet, A. 1998. Xylem colonization by an $\mathrm{HrcV}^{-}$mutant of Ralstonia solanacearum is a key factor for the efficient biological control of tomato bacterial wilt. Mol. Plant-Microbe Interact. 11:869-877.

Fåhraeus, G. 1957. The infection of clover root hairs by nodule bacteria studied by a simple glass slide technique. J. Gen. Microbiol. 16:374-381.

Geelen, D., Mergaert, P., Geremia, R. A., Goormachtig, S., Van Montgu, M., and Holsters, M. 1993. Identification of nodSUIJ genes in Nod locus 1 of Azorhizobium caulinodans: Evidence that nodS encodes a methyltransferase involved in Nod factor modification. Mol. Microbiol. 9:145-154.

Gopalaswamy, G., Kannaiyan, S., O’Callaghan, K. J., Davey, M. R., and Cocking, E. C. 2000. The xylem of rice (Oryza sativa) is colonized by Azorhizobium caulinodans. Proc. R. Soc. Lond. B. 267:103-107.
Gough, C., Webster, G., Vasse, J., Galera, C., Batchelor, C., O'Callaghan, K., Davey, M., Kothari, S., Denarie, J., and Cocking, E. C. 1996. Specific flavonoids stimulate intercellular colonization of non-legumes by Azorhizobium caulinodans. Pages 409-415 in: Biology of Plant-Microbe Interactions. G. Stacey, B. Mullin, and P. M. Gresshoff, eds. International Society for Molecular Plant-Microbe Interactions, St. Paul, MN, U.S.A.

Gough, C., Galera, C., Vasse, J., Webster, G., Cocking E. C., and Dénarié, J. 1997a. Specific flavonoids promote intercellular root colonization of Arabidopsis thaliana by Azorhizobium caulinodans ORS571. Mol. Plant-Microbe Interact. 10:560-570.

Gough, C., Vasse, J., Galera, C., Webster, G., Cocking, E. C., and Dénarié, J. 1997b. Interactions between bacterial diazotrophs and non-legume dicots: Arabidopsis thaliana as a model plant. Plant Soil 194:123-130.

Graham, T. L. 1998. Flavonoid and flavonol glycoside metabolism in Arabidopsis. Plant Physiol. Biochem. 36:135-144.

James, E. K., Reis, V. N., Olivares, F. L., Baldani, J. I., and Döbereiner, J. 1994. Infection of sugar cane by the nitrogen-fixing bacterium Acetobacter diazotrophicus. J. Exp. Bot. 45:757-766.

James, E. K., Olivares F. L., Baldani J. I., and Döberiener J. 1997. Herbaspirillum, an endophytic diazotroph colonizing vascular tissue in leaves of Sorghum bicolor L. Moench. J. Exp. Bot. 48:785-797.

Ladha, J. K., Garcia, M., Miyan, S., Padre, A. T., and Watanabe, I. 1989. Survival of Azorhizobium caulinodans in the soil and rhizosphere of wetland rice under Sesbania rostrata-rice rotation. Appl. Environ. Microbiol. 55:454-460.

Leong, S. A., Williams, P. H., and Ditta, G. S. 1985. Analysis of the $5^{\prime}$ regulatory region of the gene for $\delta$-aminolevulinic acid synthetase of Rhizobium meliloti. Nucleic Acids Res. 13:5965-5976.

Murashige, T., and Skoog, F. 1962. A revised medium for rapid growth and bioassays with tobacco tissue cultures. Physiol. Plant 15:473-497.

O'Callaghan, K. J., Davey, M. R., and Cocking, E. C. 1997. Xylem colonization of the legume Sesbania rostrata by Azorhizobium caulinodans. Proc. R. Soc. Lond. B. 264:1821-1826.

Olson, P. D., and Varner, J. E. 1993. Hydrogen peroxide and lignification. Plant J. 4:887-892.

Parniske, M., Ahlborn, B., and Werner D. 1991. Isoflavonoid-inducible resistance to the phytoalexin glyceollin in soybean rhizobia. J. Bacteriol. 173:3432-3439.

Perret, X., Fellay, R., Bjourson, A. J., Cooper, J. E., Brenner, S., and Broughton, W. J. 1994. Subtraction hybridization and shot-gun sequencing: A novel approach to identify symbiotic loci. Nucleic Acids Res. 22:1335-1341.

Reynolds, E. S. 1963. The use of lead citrate at high $\mathrm{pH}$ as an electron opaque stain in electron microscopy. J. Cell Biol. 17:208-212.

Sabry, S. R. S., Saleh, S. A., Batchelor, C. A., Jones, J., Jotham, J., Webster, G., Kothari, S. L., Davey, M. R., and Cocking, E. C. 1997. Endophytic establishment of Azorhizobium caulinodans in wheat. Proc. R. Soc. Lond. B. 264:341-346.

Scheres, B., and Wolkenfelt, H. 1998. The Arabidopsis root as a model to study plant development. Plant Physiol. Biochem. 36:21-32.

Somasegaran, P., and Hoben, H. J. 1994. Handbook for Rhizobia: Methods in Legume-Rhizobium Technology. Springer, New York.

Vasse, J., Frey, P., and Trigalet, A. 1995. Microscopic studies of intercellular infection and protoxylem invasion of tomato roots by Pseudomonas solanacearum. Mol. Plant-Microbe Interact. 8:241-251.

Webster, G., Gough, C., Vasse, J., Batchelor, C., O'Callaghan, K. J., Kothari, S. L., Davey, M. R., Dénarié, J., and Cocking, E. C. 1997. Interactions of rhizobia with rice and wheat. Plant Soil 197:115-122. 